\title{
PURIFICATION OF POLYCYCLIC AROMATIC COMPOUNDS USING SALTING-OUT SEPARATION IN HIGH-PRESSURE $\mathrm{CO}_{2}$
}

\author{
CHIEHMING J. CHANG AND YUCHUNG LIOU \\ Department of Chemical Engineering, Yuan-Ze Institute of \\ Technology, 135, Far-East Rd., Taoyuan 320, Taiwan, R.O.C.
}

Key Words: Minimum Solubility, Relative Supersaturation Ratio, Pressure-Tuning Separation, Crystallization

\begin{abstract}
Gas antisolvent crystallization has the potential for application in the recovery of valuable compounds from liquid solution, and in the separation of solid-solid mixtures. Experimental data are presented for a mixture of anthracene and anthraquinone dissolved in cyclohexanone which was expanded by a gaseous anti solvent, $\mathrm{CO}_{2}$. The pressure range is 0.1 to $12 \mathrm{MPa}$, and the temperature is 291 to $313 \mathrm{~K}$. The relation of salted-out yield and normalized feed concentration gives an important parameter, the so-called minimum solubility, from which supersaturation can be defined for gas antisolvent crystallization. Effects of initial feed concentrations of solid solutes, temperature, and pressure on the separation of anthracene and anthraquinone have also been studied.
\end{abstract}

\section{Introduction}

Separation steps are essential to virtually all manufacturing processes. In industries, thousands of products are fractionated to the desired purity by various separation techniques. Methods requiring an extraneous substance to effect the separation are usually called mass separating agent processes ${ }^{1)}$. Solution crystallization, which requires physical solvents or entrainers, is one of these processes. Separations through cooling crystallization have played a significant role in the chemical industry from its early stages ${ }^{18)}$. Even today many separations which otherwise are very difficult by chemical methods are carried out by exploiting the differences either in physical properties or solubility in liquid solvents ${ }^{1,19)}$. Crystallization with supercritical fluids is a new process of separation which is presently an active area of research ${ }^{4,5,16)}$. Many materials with high melting point and low volatility, such as pharmaceuticals, inorganics, and polymers, have only very low solubilities even in high-pressure supercritical fluids ${ }^{16)}$. For example, the solubilities of coal tar components in supercritical fluid $\mathrm{CO}_{2}$ are only around $10^{-5}$ mole fraction at $313 \mathrm{~K}$ and $15 \mathrm{MPa}^{14)}$. Low solubilities make extraction and separation of these materials using supercritical fluids unattractive.

Gas could be used as an antisolvent to reduce the strength of liquid solvent and selectively crystallize solid materials from solution. In early years this salting-out effect was proposed for the concentration of fruit juices from aqueous solutions by a gas hydrate formation clathrate type ${ }^{21)}$. Gallagher et $a .^{8)}$ were first to demonstrate that a difficult-to-comminute explosive solid, nitroguanidine, could be recrystallized from a liquid solution using compressed gas as an antisolvent. Chang and Randolph ${ }^{3)}$ then studied the solubilities of $\beta$-carotene and carotene

ReceivedFebruary 9, 1992. Correspondence concerning this article should be addressed to C. J. Chang. oxidation products in a mixed solvent expanded with $\mathrm{CO}_{2}$. They found that the content of solids in the liquid phase could be affected significantly by pressure. Their next work demonstrated that gas antisolvent crystallization could be used to separate $\beta$-carotene from carotene oxidation products and to enrich trans- $\beta$-carotene from raw $\beta$-carotene containing $\beta$-carotene isomers ${ }^{2}$. Gas antisolvent crystallization also has potential for separation and recrystallization of materials that are insoluble in supercritical fluid ${ }^{9,13)}$. They observed that at some point a critical supersaturation level is exceeded and the solid precipitates. By varying the operating conditions (temperature, pressure, initial feed concentration, and degree of solvent expansion) an effective separation of solids in solution can be achieved.

There is still little knowledge of the effect of operating conditions on separation using gas antisolvent crystallization, especially quantitative findings. From a practical point of view, the quantitative description of supersaturation as a function of pressure, temperature, and solute concentration is the most important. When two solutes are presented in a mixed solvent of gas antisolvent and liquid solvent, the determination of supersaturation of each solid solute is necessary to evaluate the efficiency of the separation. Our objective is to find a convenient representation of supersaturation of solid solute as a function of pressure, and to investigate the relation between separation efficiency and relative supersaturation ratio. Anthracene, anthraquinone, and a mixture of the two were chosen as model substances for our work. The substance is the result of the oxidation of pure anthracene obtained from filtration, vacuum distillation, and subsequent purification of the anthracene oil produced in the distillation of coal $\operatorname{tar}^{7)}$. The solubilities of these solids and high-pressure $\mathrm{CO}_{2}$ in some liquid solvents are well known ${ }^{6,10,15)}$. Those data provided valu- 

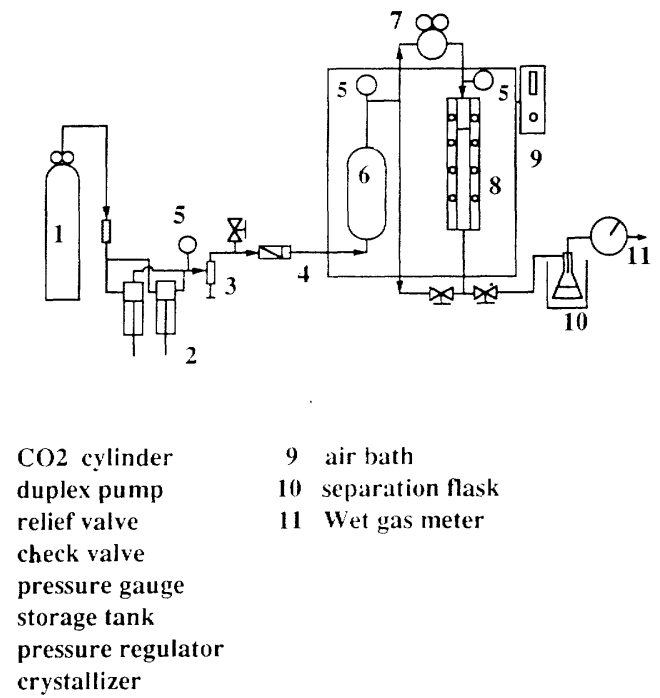

9 air bath

10 separation flask

11 Wet gas meter$$
\text { (1) }
$$

Fig. 1 Schematic flow diagram of experimental apparatus

able information for developing and evaluating the separation of solid mixtures using gas antisolvent crystallization.

\section{Experimental Section}

\subsection{Apparatus and procedure}

An experiment was designed to obtain the solvent expansion coefficient, solid samples collection after recrystallization, and mole fractions of gaseous and solid solutes in the liquid phase simultaneously. A flow cell apparatus was used to achieve this goal. A schematic flow diagram is shown in Fig. 1. The central feature of this apparatus is a salting-out vessel with a view glass (Jerguson gage, 4-T-20). For separation experiments 50 $\mathrm{mL}$ solid solute-laden liquid solution was held at the bottom of the vessel by a device consisting of three layers of hydrophobic polymer filter (Millipore, 0.45 $\mu \mathrm{m})$ and a stainless steel screen with 200-mesh grid. Liquid carbon dioxide, compressed by a high-pressure piston pump (LDC, NSI-33R), flowed into a storage vessel (Whitey, DOT-3A-1800) and attained the desired pressure. The compressed carbon dioxide was introduced into the salting-out vessel through a micro-metering valve and a device to evenly distribute gas bubbles into the solution. Pressure in the salting-out vessel was controlled by a forward pressure regulator (Go Instrument, PR-50) with an accuracy of $0.07 \mathrm{MPa}$. Temperature in the air bath was maintained within $\pm 0.1 \mathrm{~K}$ using a PI controller.

To determine the expansion coefficient of cyclohexanone and the specific liquid-phase volume of the solution when carbon dioxide was present in the liquid phase, the compressed $\mathrm{CO}_{2}$ was introduced slowly into a Jerguson gage containing $20 \mathrm{~mL}$ pure cyclohexanone, and then the liquid solution was expanded. The level of the liquid phase was measured by a standard rule and the volume of the liquid phase was calculated. Pressure recording was taken from 0.1 to $6.9 \mathrm{MPa}$ with an interval of $0.44 \mathrm{MPa}$.

Separation using gas antisolvent crystallization began with a solution of $50 \mathrm{~mL}$ cyclohexanone laden equally with anthracene and anthraquinone. During crystallization the system was allowed to equilibrate for 30 minutes, while anthracene and anthraquinone crystals could be seen on the surface of the view glass. After crystallization, under the same operating pressure the solution was depleted through the bottom of the crystallizer. At the same time fresh liquid carbon dioxide, introduced from the top of the cell, maintained pressure, washed and dried the crystallized solids for a fixed period. The purpose of this top-down washing is to take away solvent residual included inside solid crystallites. There is little re-dissolution of crystals in the $\mathrm{CO}_{2}$ according to the study of solubilities of anthracene in SF $\mathrm{CO}_{2}$ by Lisicki et al. ${ }^{14)}$ The mole fraction of anthracene in high-pressure $\mathrm{CO}_{2}$ (i.e. 200 bars and $40^{\circ} \mathrm{C}$ ) is 2.0 $(10)^{-5}$. Compared to the amount salted-out, the error due to re-dissolution of solids in the high-pressure $\mathrm{CO}_{2}$ could be neglected in the process of gas antisolvent crystallization. In the period of crystallization and washing, total pressure drop was less than $0.07 \mathrm{MPa}$ because of stabilization by a feed-forward pressure controller. De-pressurized solution and carbon dioxide separated and collected in a separation flask. The amount of carbon dioxide used in the whole process was obtained from a wet-gas meter (Shinagawa, W-NK-1A).

\subsection{Reagents and analysis}

In the quantitative analysis of liquid samples, we used a reference standard method. Therefore, anthracene (Merck, 96+\%), anthraquinone (Merck, 98+\%), and analytical-grade solvents such as methanol and cyclohexanone were used as received without need of purification. Carbazole (Merck, 98+\%) was used as an internal reference standard for quantitative analysis of the solid solutes. Concentrations of solid solutes in each solution were analyzed by a high-performance liquid chromatograph (HPLC). The HPLC (Waters, Model 510), equipped with a UV detector, utilized a reverse phase column (Novapak, C-18) with a mobile phase containing $82 \%(\mathrm{v} / \mathrm{v})$ methanol and $18 \%(\mathrm{v} / \mathrm{v})$ water at a flow rate of $1.2 \mathrm{~mL} / \mathrm{min}$. Through quantitative analysis of solid-solute concentrations of solutions before and after gas antisolvent crystallization, yields and solid solutes retained in the depleted solution at each pressure were obtained from a mass balance calculation.

Once the determination of solid content in the depleted solution was made, mole fractions of components (i.e. $\mathrm{CO}_{2}$, anthracene, and anthraquinone) in the liquid phase were calculated from a known amount of $\mathrm{CO}_{2}$ dissolved in the liquid phase, which could be obtained from the difference between total moles of $\mathrm{CO}_{2}$ charged into the cell and moles of $\mathrm{CO}_{2}$ in the vapor phase. The procedure for this calculation was shown elsewhere ${ }^{11)}$. Typical uncertainties of mole fractions in the liquid phase were $8 \%$, including the loss of cyclo- 


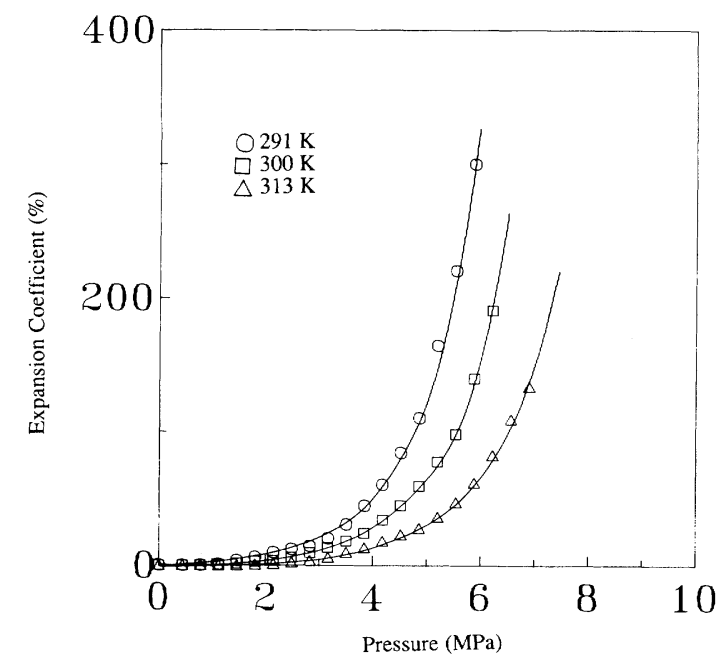

Fig. 2 Volume expansion coefficients of cyclohexanone with $\mathrm{CO}_{2}$ at 291,300 , and $313 \mathrm{~K}$

hexanone vapor carried over with $\mathrm{CO}_{2}$ flow during depletion of the expanded solution and the error in measurement of liquid- and vapor-phase volumes. The specific volume of the system was measured by using a calibration technique as a product of the mass of $\mathrm{CO}_{2}$ collected at atmospheric pressure and the reciprocal of the density of high-pressure $\mathrm{CO}_{2}$ charged into the crystallizer. Liquid volume of the solution was computed from an expansion curve of $\mathrm{CO}_{2}$ in cyclohexanone. The uncertainty is estimated to be less than $5 \%$.

Solubilities of anthracene and anthraquinone in cyclohexanone at constant temperature were determined by HPLC analysis. The equilibrium experiments were carried out in a constant-temperature air-bath shaker (Firstec Scientific, S300R) which was controlled at \pm 0.1 $\mathrm{K}$. The shaker was continuously in operation for at least 12 hours. Table 1 lists physical properties of compounds required for the calculation.

\section{Results and Discussion}

The expansion coefficient of the solvent, which is a function of temperature and pressure, is defined as

$$
\Phi=\left(\frac{V^{L}(T, P)}{V^{\circ}(T, 0.1 \mathrm{MPa})}-1\right) \times 100 \%
$$

Figure 2 shows three expansion isotherms of cyclohexanone with $\mathrm{CO}_{2}$ at 291, 300, and $313 \mathrm{~K}$, respectively. Cyclohexanone was expanded by carbon dioxide slightly at lower pressure but tremendously at higher pressure. The expansion of cyclohexanone saturated with $\mathrm{CO}_{2}$ increased as temperature decreased.

Solid solubility in the liquid solvent is essential in the study of the separation process. Usually, the solid dissolves in an organic solvent to form a solution, and the difference between the ideal and real solutions can give useful information about interactions between solute and solvent. Ideal solubilities for both anthracene

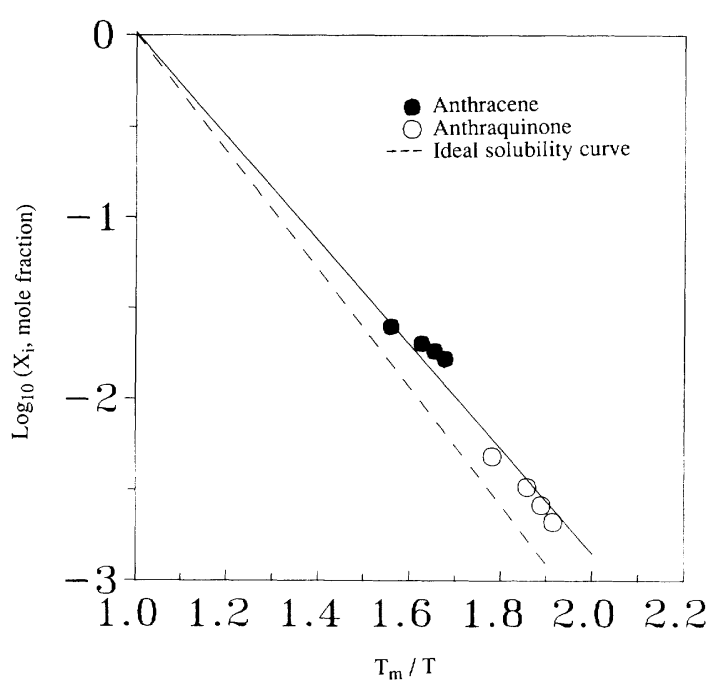

Fig. 3 Comparison of experimental and ideal solubilities of anthracene and anthraquinone in cyclohexanone at 291, 295,300 , and $313 \mathrm{~K}$

and anthraquinone in cyclohexanone can be given by the following equation ${ }^{17}$.

$$
\operatorname{Ln} X_{i}=\frac{\Delta H_{f}}{R T_{m}}\left(\frac{T_{m}}{T}-1\right)
$$

Experimental and ideal solubilities of anthracene and anthraquinone are shown in Fig. 3. In the range of temperature measured the experimental solubilities are higher than the calculated values for both solids, which indicates positive deviations from ideal solutions. The solid line represents the average solubilities of the real solutions. On one isotherm, the solubility of anthracene is about five times that of anthraquinone.

\subsection{Minimum solubility and supersaturation in gas antisolvent crystallization}

Nucleation occurs at the point where solute concentration exceeds the value of its solubility in the solution. The supersaturation is the common term for quantitatively showing the distance of the solute from its nucleation point. In gas antisolvent crystallization, anthracene and anthraquinone were co-precipitated from the solution expanded with high-pressure $\mathrm{CO}_{2}$. To create an even opportunity for both solutes to nucleate in the solution, an equal degree of saturation was initially prepared for anthracene and anthraquinone to form socalled feed solutions containing the same degree of saturation for both solid solutes. The yields of anthracene and anthraquinone were simultaneously obtained at each isobaric curve for 291 and $313 \mathrm{~K}$, respectively. The yield in weight fraction, $\mathrm{Y}$, can be defined as the ratio of the amount of salted-out solute from a solution at a certain pressure to that of solute contained in the feed solution at atmospheric pressure. We adopted the normalized feed concentration, which is defined as the feed concentration of each solid solute divided by the solubility of that solute at atmospheric pressure. The relation of the exper- 


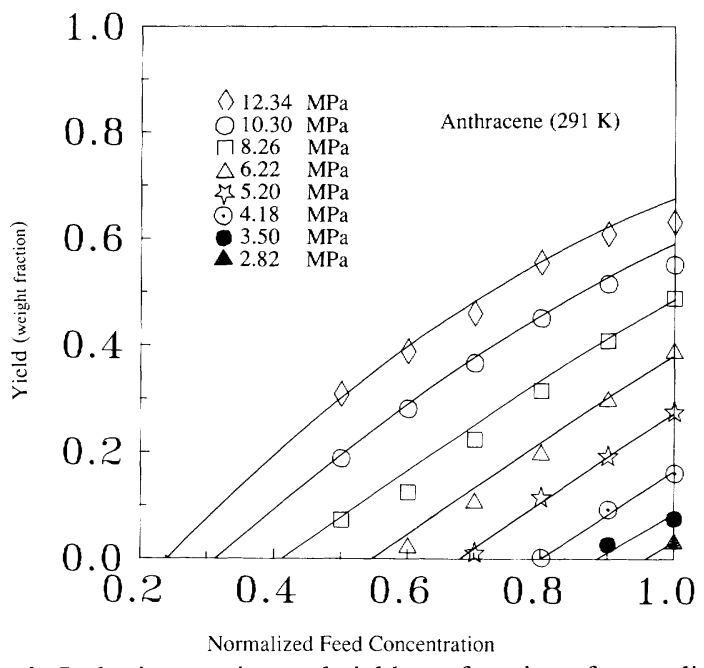

Fig. 4 Isobaric experimental yield as a function of normalized feed concentration of anthracene at $291 \mathrm{~K}$

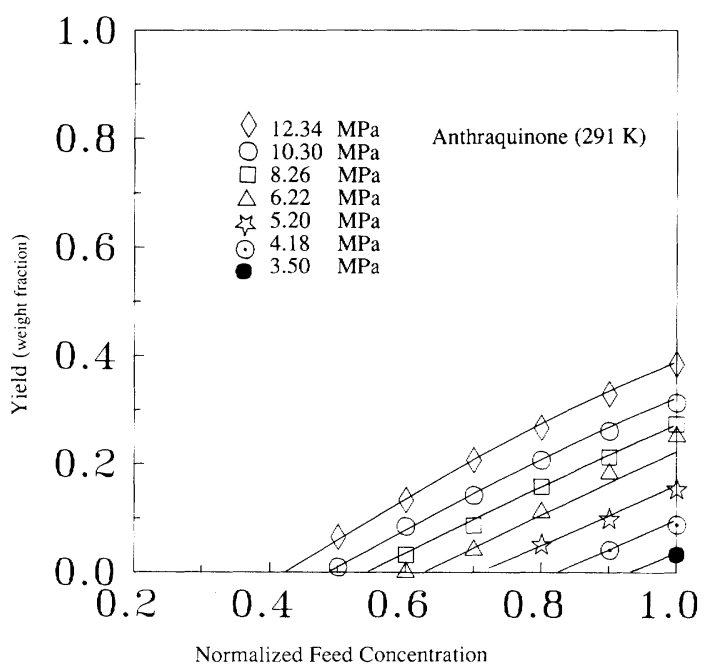

Fig. 5 Isobaric experimental yield as a function of normalized feed concentration of anthraquinone at $291 \mathrm{~K}$

imental yields to the normalized feed concentrations at $291 \mathrm{~K}$ as shown in Figs. 4 and 5. It can be seen that the yield of anthracene is larger than the yield of anthraquinone at the same level of expansion. The yield increases with normalized feed concentration. Using a leastsquares method we extrapolated the experimental yield to the zero value of each isobaric curve. The extrapolated point in the abscissa is the point where nucleation starts at a given pressure. We define it as the hypothetical "minimum solubility" of the solid solute at a given pressure and temperature. The addition of gas antisolvent will increase the concentration of solid solute beyond its minimum solubility and cause precipitation. The minimum solubility of one solid solute therefore is a function of pressure when gas antisolvent crystallization operated at constant temperature. A hypothetical supersaturation of one solute might be obtainable in the following form:

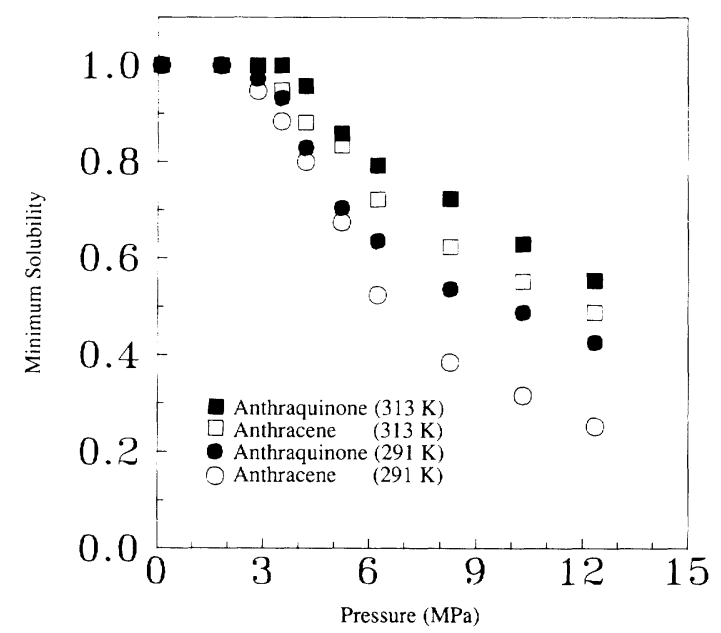

Fig. 6 Minimum solubility of solid solute in gas antisolvent crystallization of $\mathrm{CO}_{2}$ cyclohexanone-anthraceneanthraquinone at 291 and $313 \mathrm{~K}$

$$
S S(P)=\frac{C(0.1 \mathrm{MPa})-C_{\text {sat }}(P)}{C_{\text {sal }}(P)}
$$

When gas antisolvent crystallization operates at constant temperature, this hypothetical definition indicates that supersaturation is pressure-dependent. Supersaturation at atmospheric pressure equals zero. The increase of solid-solute concentration due to a certain degree of expansion level results in the precipitation of solid solute from the solution. To better identify which solute precipitated more than the other solute in gas antisolvent crystallization, the minimum solubility of each solute plotted against pressure at 291 and $313 \mathrm{~K}$ is shown in Fig. 6. In the whole range of pressure investigated, anthraquinone was identified to have larger minimum solubilities than anthracene. In other words, anthraquinone needs a higher expansion level to precipitate than anthracene does. This implied that at the same expansion level anthraquinone will not precipitate but anthracene will. It is also consistent with the point that gas antisolvent crystallization at $313 \mathrm{~K}$, which has larger minimum solubility, needs a higher expansion level to salt-out solid solutes from the solution than does low-temperature crystallization. This explanation using minimum solubility gave better insight into gas antisolvent crystallization than that using mole fractions of solid solutes in the liquid phase ${ }^{3,6)}$.

\subsection{Separation factor and relative supersaturation ratio}

To predict more quantitatively the separation of two solid solutes in gas antisolvent crystallization, we evaluated its efficiency by the separation factor, $\alpha$, rather than by the solid-solute solubility in the liquid phase. The separation factor $(\alpha)$ of one solute to the other solute could be obtained from the ratio $\left(Y_{i} / Y_{i}\right)$ of salted-out yields of these two solid solutes. To map out the region available for the separation of anthracene and anthraquinone, we adopted five solutions containing both solids in 


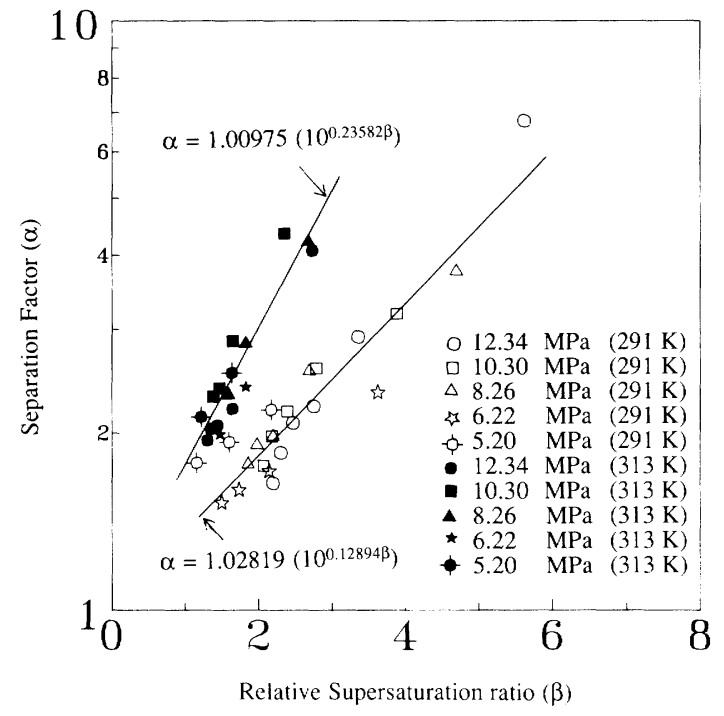

Fig. 7 Separation factor correlated with relative supersaturation ratio of two solids in gas antisolvent crystallization of $\mathrm{CO}_{2}$ cyclohexanone-anthraceneanthraquinone at 291 and $313 \mathrm{~K}$

cyclohexanone with normalized feed concentration from 0.5 up to 1.0. Then, the separation factor of anthracene to anthraquinone represents the actual separation efficiency in gas antisolvent crystallization without the effect of solubility enhancement by the presence with the other solid in the solution ${ }^{12)}$.

To further visualize the effect of operating parameters on the separation of anthracene and anthraquinone, it is informative to define a quantitative value for illustrating the degree of potential precipitation of one solute over the other, that is, the relative supersaturation ratio, which can be expressed as

$$
\beta=\frac{S S(P) \text { anthracene }}{S S(P) \text { anthraquinone }}
$$

Unlike cooling crystallization controlled by temperature change, the driving force (i.e. supersaturation) for inducing precipitation in gas antisolvent crystallization depends on pressure. A plot of separation factor, $\alpha$, versus relative supersaturation ratio, $\beta$, for all investigated pressures is shown in Fig. 7. In this figure the blank and the dark symbols represent experimental data at 291 and $313 \mathrm{~K}$, respectively. All isobars seem to be linear upward, indicating that separation factors of anthracene versus anthraquinone could be readily obtained from their relative supersaturation ratio at each normalized feed concentration, temperature, and pressure. Straight lines drawn by linear regression for both temperatures studied showed that high temperature (313 K) gave better separation than low temperature (291 K), when operating at the same relative supersaturation ratio. This might be qualitatively explained in a simple manner in that the similarity among anthraquinone, cyclohexanone, and $\mathrm{CO}_{2}$ makes anthraquinone more likely to remain in the solution than anthracene. Hence the
Table 1. Physical properties of compounds used in separation study

\begin{tabular}{lccc}
\hline compound & anthracene & anthraquinone & cyclohexanone \\
\hline$T_{m}(\mathrm{~K})^{*}$ & 483.86 & 555.34 & - \\
$\Delta H_{f}(\mathrm{cal} / \mathrm{g})^{*}$ & 39.93 & 39.98 & - \\
$M W(\mathrm{~g} / \mathrm{moL})^{* *}$ & 178.0 & 208.0 & 98.0 \\
$\rho(\mathrm{g} / \mathrm{mL})^{* *}$ & 1.283 & 1.430 & 0.950 \\
$S\left(\mathrm{kG} / \mathrm{m}^{3}\right)^{*}$ & & & \\
$291 \mathrm{~K}$ & 29.112 & 4.256 & - \\
$295 \mathrm{~K}$ & 31.886 & 5.218 & - \\
$300 \mathrm{~K}$ & 35.066 & 6.572 & - \\
$313 \mathrm{~K}$ & 43.638 & 9.690 & - \\
\hline \multirow{2}{*}{ this study } & & & \\
**Weast and Grasselli ${ }^{20)}$ & &
\end{tabular}

decrease in the solubility parameter at $313 \mathrm{~K}$ affects anthraquinone less than anthracene.

Based upon the discussion of the effects of temperature, pressure, and normalized feed concentration on the separation in gas antisolvent crystallization, the mechanism for the separation could be readily understood by the correlation between separation factor and relative supersaturation ratio of solid solutes. The massseparating agent in gas antisolvent crystallization is a mixture of a gas antisolvent and a liquid solvent. With this antisolvent addition and removal controlled by adjustment of the system pressure, the recovery of pure anthracene from the solution could be simply achieved by membrane filtration without any solid anthraquinone being present. Many other easier paths can be devised by beginning at different normalized feed concentrations and by manipulating pressure and temperature.

\section{Conclusions}

The experimental flow-cell technique provides a means of measuring the specific liquid-phase volume and rapidly achieving the desired separation. In gas antisolvent crystallization, minimum solubility of solid solute has been experimentally determined from its salted-out yield. Supersaturation which uses pressure as the adjustable parameter could be adopted to represent the driving force for solids precipitation in the process. The relative supersaturation ratio revealed that anthracene, having a relatively low minimum solubility, would precipitate first and mainly. Relative supersaturation ratios have been correlated with separation factors. The discussion of operating parameters on the separation also disclosed that gas antisolvent crystallization can potentially be used to separate some solid-solid mixtures by easily varying the normalized feed concentration of each solute, the amount of $\mathrm{CO}_{2}$ addition (i.e. pressure), and temperature. It was demonstrated that gas antisolvent crystallization provides more flexible parameters 
for separating one solid component from the other, especially for those mixtures having a large difference in melting temperature, when compared to thermally driven crystallization.

\section{Acknowledgment}

The authors gratefully acknowledge the financial support of this work by the National Science Council of ROC under Grant No. NSC810402-E155-514.

\section{Nomenclature}

\begin{tabular}{|c|c|c|c|}
\hline$C_{s a t}(\mathrm{P})$ & $=$ & minimum solubility of solid solute & \\
\hline$C(0.1 \mathrm{MPa})$ & $=$ & normalized feed concentration of solid solute & \\
\hline$\Delta H_{f}$ & $=$ & heat of fusion at normal melting temperature & {$[\mathrm{cal} /$} \\
\hline$M W$ & $=$ & molecular weight & [g/mole] \\
\hline$P$ & $=$ & pressure & {$[\mathrm{MP}$} \\
\hline$R$ & $=$ & gas constant $(=8.314)$ & $\mathrm{J} / \mathrm{mole} / \mathrm{K}]$ \\
\hline$S$ & $=$ & solubility of solid solute in liquid solvent & {$\left[\mathrm{kg} / \mathrm{m}^{3}\right]$} \\
\hline$S S(\mathrm{P})$ & $=$ & supersaturation of solid solute & \\
\hline$T_{m}$ & $=$ & normal melting temperature & \\
\hline$V$ & $=$ & specific volume of solution & {$[\mathrm{c}$} \\
\hline$X_{i}$ & $=$ & mole fraction of component $i$ in liquid phase & \\
\hline$Y_{i}$ & $=$ & yield of solid $i$ in salting out, weight fraction & \\
\hline$\alpha$ & $=$ & separation factor & \\
\hline$\beta$ & $=$ & relative supersaturation ratio & \\
\hline$\Phi$ & $=$ & expansion coefficient & \\
\hline$\rho$ & $=$ & density & {$[\mathrm{g} / \mathrm{c}$} \\
\hline
\end{tabular}

$<$ Superscripts>

$L \quad=$ liquid phase

$\therefore \quad=$ reference state

Literature Cited

1) Barnicki, S.D. and J.R. Fair: Ind. Eng. Chem. Res., 29, 421-432 (1990)

2) Chang, C.J., A.D. Randolph and N.E. Craft: Biotechnol. Prog., 7, 275-278 (1991)

3) Chang, C.J. and A.D. Randolph: AIChE J., 36, 939-942 (1990)

4) Chimowitz, E.H. and K.J. Pennisi: AIChE J., 32,1665-1672 (1986)
5) Dhalewadikar, S.V., A.J. Seckner and M.A. McHugh: Ind. Eng. Chem. Res., 26, 976-982 (1987)

6) Dixon, D.J. and K.P. Johnston: AIChE J., 37, 1441-1449 (1991)

7) Frank, H.G. and J.W. Stadelhofer: "Industrial Aromatic chemistry," Chapter 3, Spring-Verlag, Berlin (1988)

8) Gallagher, P.M., M.P. Coffey, V.J. Krukonis and N. Klasuties: Amer. Chem. Soc. Symp. Ser., No. 406, p. 334-354 (1989)

9) Gallagher, P.M., V.J. Krukonis and L.J. Vandekieft: Proceedings of 2nd Int. Symp. on Supercritical Fluids, M.A. McHugh, Ed., Boston, MA (1991)

10) Gurevich, B.S., E.M. Sizova and M.M. Isaenko: Koks Khimiya, 1, 36-38 (1977)

11) Kato, M., K. Aizawa, T. Kanahira and T. Ozawa: J. Chem. Eng. Japan, 24, 767-771 (1991)

12) Kurnik, R.T. and R.C. Reid: Fluid Phase Equil., 8, 93-105 (1982)

13) Liou Y. and C.J. Chang: Sep. Sci. and Technol., 27, 1277-1289 (1992)

14) Lisicki, Z., W. Majewski, J. Kwiatkowski, A. Dunalewicz and J. Polaczek: Fuel Processing Technology, 20, 103-121 (1988)

15) Litvinenko, M.S., L.D. Gluzman, A.A. Rok, S.N. Kipot, V.P. Bogunets, N.I. Zhuravskaya, R.M. Zil'berman and L.I. Didenko: Koks Khimiya, 3, 33-36 (1976)

16) McHugh, M.A. and V.J. Krukonis: "Supercritical Fluid Extraction: Principles and Practices," Chapter 12, Butterworths, MA (1986)

17) Prausnitz, J.M., R.N. Nichtenthaler and E.G. de Azevedo: "Molecular Thermodynamics of Fluid Phase Equilibria," Chapter 9, Prentice-Hall, Englewoods Cliffs, NJ (1986)

18) Randolph, A.D. and M.A. Larson: "Theory of Particulate Processes: Analysis and Techniques of Continuous Crystallization," 2nd Ed., Chapter 5, Academic Press, CA (1988)

19) Rodriguez, F., L.F. Adrados, J.C. Burillo and J.F. Tijero: Analyst, October, 114, 1241-1244 (1989)

20) Weast R.C. and J.G. Grasselli: "Handbook of Data on Organic Compounds," 2nd Ed., Vol. I, CRC press Inc., Boca Raton, FL (1989)

21) Werezak, G.N.: Amer. Chem. Soc. Symp. Ser. No. 65 ,6-18 (1969) 